\title{
AHNAK is highly expressed and plays a key role in cell migration and invasion in mesothelioma
}

\author{
HITOMI SUDO ${ }^{1}$, ATSUSHI B. TSUJI ${ }^{1}$, AYA SUGYO $^{1}$, MASAAKI ABE $^{2}$, OKIO HINO $^{2}$ and TSUNEO SAGA $^{1}$ \\ ${ }^{1}$ Diagnostic Imaging Program, Molecular Imaging Center, National Institute of Radiological Sciences, \\ Inage-ku, Chiba 263-8555; ${ }^{2}$ Department of Pathology and Oncology, Juntendo University \\ School of Medicine, Bunkyo-ku, Tokyo 113-8421, Japan
}

Received August 27, 2013; Accepted October 10, 2013

DOI: $10.3892 /$ ijo.2013.2183

\begin{abstract}
The worldwide incidence of the highly aggressive tumor mesothelioma is expected to increase. Mesothelioma is classified into three main histological subtypes: epithelioid, sarcomatoid and biphasic. Although the pathological diagnostic markers for epithelioid are established, to date no adequate marker for sarcomatoid mesothelioma has been found. Thus, a reliable diagnostic marker of sarcomatoid mesothelioma is necessary. In this study, to identify an unknown protein with $120 \mathrm{kDa}$ expressed only in the mesothelioma cell line $211 \mathrm{H}$, we conducted proteomic analysis and found five candidate proteins. One such protein, AHNAK, was highly expressed in all seven mesothelioma cell lines $(211 \mathrm{H}, \mathrm{H} 28$, H226, H2052, H2452, MESO1 and MESO4), but not in the mesothelial cell line MeT-5A by RT-PCR and immunofluorescence staining. Furthermore, we confirmed high AHNAK expression not only in xenografts but also in human mesothelioma specimens including sarcomatoid, epithelioid and biphasic mesothelioma using immunohistochemical staining. These findings suggest that AHNAK has the potential to be a new marker for detecting mesothelioma. Since AHNAK is involved in cell migration and invasion in other metastatic tumor cells, we conducted migration and invasion assays in mesothelioma cell lines. The number of migrating cells in six of seven mesothelioma cell lines and the number of invading cells in all seven cell lines were significantly increased compared with those in MeT-5A. Knockdown of AHNAK significantly reduced the cell migration and invasion ability in all seven mesothelioma cell lines. These results support further clinical evaluation of the association of AHNAK and metastasis in mesothelioma.
\end{abstract}

Correspondence to: Dr Atsushi B. Tsuji, Diagnostic Imaging Program, Molecular Imaging Center, National Institute of Radiological Sciences, 4-9-1 Anagawa, Inage-ku, Chiba 263-8555, Japan E-mail: a_tsuji@nirs.go.jp

Key words: mesothelioma, immunohistochemical staining, migration, invasion

\section{Introduction}

Malignant mesothelioma, a highly aggressive tumor which arises from mesothelial cells on serosal surfaces, is most commonly seen in the pleura, followed by the peritoneum, pericardium and male genitalia $(1,2)$. Mesothelioma formation is mostly attributed to asbestos exposure, and the lag time between exposure and disease is 30-40 years (3). Although mesothelioma has been considered to be a rare tumor, its incidence is anticipated to increase globally $(3,4)$. Malignant mesothelioma has a poor prognosis; a large population-based study reported that 6-month, 1-year, and 5-year overall survival rates were 55,33 , and $5 \%$, respectively (5). The median survival of mesothelioma has been reported to be 9-10 months from the time of diagnosis (6). In the classification based on histological categories, three main types of mesothelioma are reported: epithelioid, sarcomatoid and biphasic. The epithelioid subtype is the most common and it has a better prognosis than the sarcomatoid and biphasic subtypes. Several markers for epithelioid subtype, such as podoplanin, calretinin, WT-1, cytokeratin 5, thrombomodulin and ERC/mesothelin, are widely used in the clinical diagnosis of epithelioid mesothelioma $(7,8)$. However, as no adequate marker is currently available for sarcomatoid mesothelioma, accurate diagnosis of the sarcomatoid subtype is difficult. As sarcomatoid mesothelioma rarely responds to any of the currently available treatments, development of a new effective treatment is awaited. To precisely evaluate the efficacy of new types of therapy, an accurate diagnosis is essential. Immunohistochemistry of specific markers in cancer specimens plays an important role in the definitive diagnosis. Therefore, there is a critical need to determine a new reliable marker for sarcomatoid mesothelioma.

We previously reported that coatomer protein complex, subunit $\alpha$ (COPA) is highly expressed not only in epithelioid but also in sarcomatoid mesothelioma, suggesting that it is a candidate protein as a new marker for the diagnosis of sarcomatoid (9). Unfortunately, there was no suitable antibody against COPA for immunohistochemical staining. To develop a new anti-COPA antibody applicable to immunohistochemistry, we made four antibodies by immunizing rabbits with four types of synthetic peptides and evaluated each one as a candidate antibody for the immunohistochemical staining of COPA, however, none of these antibodies was suitable. Unexpectedly, 
one of these antibodies detected an unknown protein of $120 \mathrm{kDa}$ that was expressed only in the membrane fraction of the mesothelioma cell line $211 \mathrm{H}$, which formed a sarcomatoid tumor; this protein was not expressed in the mesothelial cell line MeT-5A by western blotting. This unknown protein was considered to be expressed specifically in mesothelioma and to have the potential to serve as a new molecular marker for sarcomatoid mesothelioma. In this study, to characterize this unknown protein, we conducted proteomic analysis of the membrane fraction of $211 \mathrm{H}$ and MeT-5A cells, and identified that the protein is AHNAK. Then, the expression of AHNAK was evaluated in mesothelioma cell lines, xenografts and human specimens. Furthermore, we investigated whether AHNAK is associated with migration and/or invasion in mesothelioma cell lines.

\section{Materials and methods}

Cell culture. We obtained five human mesothelioma cell lines (211H, H28, H226, H2052, and H2452) and the human mesothelial cell line MeT-5A from American Type Culture Collections (Manassas, VA, USA). Two human mesothelioma cell lines ACC-MESO-1 (MESO1) and ACC-MESO-4 (MESO4) were obtained from RIKEN Cell Bank (Tsukuba, Japan). The cells were maintained in RPMI-1640 medium supplemented with 5\% FBS (JRH Biosciences, Lenexa, KS, USA) in a humidified incubator maintained at $37^{\circ} \mathrm{C}$ with $5 \% \mathrm{CO}_{2}$.

Western blotting. We obtained an anti-COPA antibody by immunizing rabbits with the synthetic peptide CAQFHPTEDL VVSASLDQTV that is a component part of human COPA. The membrane and cytoplasmic protein fractions were separated using a ProteoExtract Transmembrane Protein Extraction kit (Merck Millipore, Billerica, MA, USA). Western blotting was conducted using anti-COPA or anti- $\beta$-actin antibody (Sigma).

Proteomic analysis. The unknown protein band was detected by western blotting using the anti-COPA antibody, and gel pieces of the corresponding size were excised. Proteins in the gel pieces were analyzed by a liquid chromatographytandem mass spectrometry (LC-MS/MS) system and mass spectrometry data were processed to provide protein identifications using the non-redundant NCBI protein database with MASCOT software (Matrix Science, Boston, MA, USA). LC-MS/MS analysis and database search were conducted by APRO Science (Tokushima, Japan).

Quantitative real-time RT-PCR. We synthesized first-strand cDNAs from the cells using a FastLane Cell cDNA kit (Qiagen, Hilden, Germany). Predesigned and preoptimized TaqMan probes to detect AHNAK, non-erythroid $\alpha$-spectrin, and $18 \mathrm{~S}$ rRNA were purchased from Applied Biosystems (Foster City, CA, USA). Real-time RT-PCR was conducted in triplicate using a Premix Ex Taq reagent (Takara Bio, Otsu, Japan). Gene expression levels were normalized to $18 \mathrm{~S}$ rRNA expression in each sample. Three independent experiments were conducted.

Immunofluorescence staining. Cells were grown on glass coverslips and fixed with $4 \%$ paraformaldehyde. Immunofluorescence staining was conducted using anti-AHNAK
(Abcom, Cambridge, UK) and Alexa Fluor 594 goat antimouse antibodies (Molecular Probes, Eugene, OR, USA). The coverslips were mounted in mounting medium with DAPI (Vector Laboratories, Burlingame, CA, USA). Fluorescence images were captured with an exposure time of $1 / 1.5 \mathrm{sec}$ for AHNAK.

Mesothelioma xenografts. The animal experiment was approved by the Institutional Animal Care and Use Committee of the National Institute of Radiological Sciences. Mesothelioma xenografts were established by injecting mesothelioma cells subcutaneously into the flanks of BALB/c-nu/nu male mice (CLEA Japan, Tokyo, Japan).

Human specimens. Mesothelioma tissue specimens were resected from 10 patients (six sarcomatoid, two epithelioid, and two biphasic subtypes) in Juntendo University Hospital. This study was approved by the Institutional Review Boards of Juntendo University School of Medicine and the National Institute of Radiological Sciences.

Immunohistological analysis. Xenografted tumors and human specimens were fixed in $10 \%$ neutral buffered formalin and embedded in paraffin for sectioning. The tissue sections were stained with the anti-AHNAK or anti-ERC/mesothelin (22A31) (10) antibody and counterstained with hematoxylin. The adjacent sections were stained with hematoxylin-eosin (H\&E) staining.

Transfection of siRNA. Stealth RNAi siRNAs for AHNAK and negative control were purchased from Applied Biosystems. The cells were transfected with $50 \mathrm{nM}$ siRNA using Lipofectamine 2000 transfection reagent (Invitrogen, Carlsbad, CA, USA). Forty-eight hours after transfection, the knockdown efficiency was confirmed by quantitative real-time PCR as mentioned above.

Cell migration and invasion assays. Migration and invasion assays were conducted in 24-well plates using $8-\mu \mathrm{m}$ pore size inserts (Becton-Dickinson Bioscience, Bedford, MA, USA) without Matrigel coating (for the migration assay) and with Matrigel coating (for the invasion assay). The inserts were placed into the wells of the 24-well plates containing RPMI-1640 with $10 \%$ FBS. Cells (5x10 4 cells) in RPMI-1640 with $1 \%$ FBS were seeded to the inserts. After $22 \mathrm{~h}$, nonmigrating and non-invasive cells were removed from the top of the filter, and migrating cells and invasive cells on the bottom of the filter were fixed with $100 \%$ methanol and stained with $0.125 \%$ crystal violet (Wako Pure Chemical Industries). The cells were counted in five randomly selected fields at x200 magnification.

Statistical analysis. The data were statistically analyzed by ANOVA with the Dunnett's multiple comparison test, or by Student's t-test.

\section{Results}

Identification of the unknown protein expressed only in the membrane fraction of $211 \mathrm{H}$ cells. Western blotting with the 


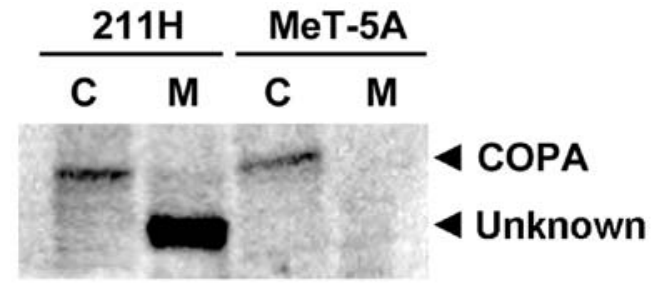

Figure 1. Western blot analysis using the anti-COPA antibody. Western blot analyses of cytosolic (C) and membrane (M) fractions of the mesothelioma cell line $211 \mathrm{H}$ and the mesothelial cell line MeT-5A were performed using the anti-COPA antibody.

anti-COPA antibody for membrane and cytosolic protein fractions of $211 \mathrm{H}$ and MeT-5A cells detected a protein band with the same molecular weight as COPA $(140 \mathrm{kDa})$ in the cytosolic fraction of $211 \mathrm{H}$ and MeT-5A cells (Fig. 1). In addition, another protein band with a smaller molecular weight $(120 \mathrm{kDa})$ compared to COPA was detected only in the membrane fraction of $211 \mathrm{H}$ cells (Fig. 1). To explore whether this protein band is a fragment of COPA or a different protein, the piece corresponding to the protein band of $120 \mathrm{kDa}$ was excised from the gel and subjected to LC-MS/MS analysis. According to the results of a protein database search of mass spectrometry data, the peptides in the gel piece of $211 \mathrm{H}$ and MeT-5A cells were matched to nine and 12 proteins, respectively. The identified proteins are listed in Table I with their accession number (GI no.), name, total score and matched peptide number. Four proteins (talin, fatty acid synthase, filamin A and nuclear pore complex protein Nup214) were detected in both $211 \mathrm{H}$ and MeT-5A cells. Although filamin A $\alpha$ (actin binding protein 280), isoform CRA_a in $211 \mathrm{H}$ cells, and filamin-A isoform 1 in MeT-5A cells, have a distinct accession number (119593150 and 116063573, respectively), these two proteins are isoforms of filamin $\mathrm{A}$ and these amino acid sequences are $99.5 \%$ identical. Five other proteins (neuroblast differentiation-associated protein AHNAK, non-erythroid $\alpha$-spectrin, Treacher Collins syndrome, pro-ubiquitin and protein BAT2like 2 ) were identified only in $211 \mathrm{H}$ cells. The remaining eight proteins $(\beta$-spectrin, spectrin- $\alpha, \beta$-filamin, non-muscle myosin heavy chain, retinoid-acid induced protein 1, plectin, NUP210 protein, and eukaryotic protein synthesis initiation factor) were identified only in MeT-5A cells. There was no peptide sequence matching COPA. Between the five proteins identified only in $211 \mathrm{H}$ cells, the total score and matched peptide number of AHNAK (total score, 1,186; matched peptide number, 24) were markedly higher than those of the others. Although AHNAK and non-erythroid $\alpha$-spectrin were matched by multiple peptides ( 24 and 5 peptides, respectively), three other proteins (Treacher Collins syndrome, pro-ubiquitin, and protein BAT2-like 2) were matched by only one peptide.

AHNAK expression analysis in cultured cells derived from human mesothelioma. The mRNA expression of AHNAK and non-erythroid $\alpha$-spectrin in mesothelioma and mesothelial cell

Table I. The identified proteins.

\begin{tabular}{|c|c|c|c|c|}
\hline Cell line & $\begin{array}{l}\text { Accession no. } \\
\text { (GI no.) }\end{array}$ & Protein name & $\begin{array}{l}\text { Total } \\
\text { score }\end{array}$ & $\begin{array}{l}\text { No. of } \\
\text { peptide }\end{array}$ \\
\hline \multirow[t]{9}{*}{$211 \mathrm{H}$} & 6739602 & Talin & 1732 & 37 \\
\hline & 61743954 & Neuroblast differentiation-associated protein AHNAK isoform 1 & 1186 & 24 \\
\hline & 38648667 & fatty acid synthase & 585 & 12 \\
\hline & 119593150 & Filamin A, $\alpha$ (actin binding protein 280), isoform CRA_a & 258 & 7 \\
\hline & 33946327 & Neuclear pore complex protein Nup214 & 181 & 6 \\
\hline & 179106 & Non-erythroid $\alpha$-spectrin & 150 & 5 \\
\hline & 1778432 & Treacher Collins syndrome & 57 & 1 \\
\hline & 340062 & Pro-ubiquitin & 55 & 1 \\
\hline & 115298682 & Protein BAT2-like 2 & 45 & 1 \\
\hline \multirow[t]{12}{*}{ MeT-5A } & 338443 & $\beta$-spectrin & 1403 & 30 \\
\hline & 62089306 & Spectrin, $\alpha$, non-erythrocytic 1 variant & 1291 & 23 \\
\hline & 6739602 & Talin & 837 & 15 \\
\hline & 116063573 & Filamin-A isoform 1 & 635 & 17 \\
\hline & 3298597 & $\beta$-filamin & 197 & 5 \\
\hline & 38648667 & Fatty acid synthase & 161 & 3 \\
\hline & 33946327 & Neuclear pore complex protein Nup214 & 120 & 3 \\
\hline & 189036 & Non-muscle myosin heavy chain (NMHC) & 109 & 1 \\
\hline & 12053793 & Retinoid-acid induced protein 1 & 96 & 1 \\
\hline & 1296662 & Plectin & 68 & 1 \\
\hline & 45595564 & NUP210 protein & 65 & 1 \\
\hline & 3941724 & Eukaryotic protein synthesis initiation factor & 54 & 1 \\
\hline
\end{tabular}




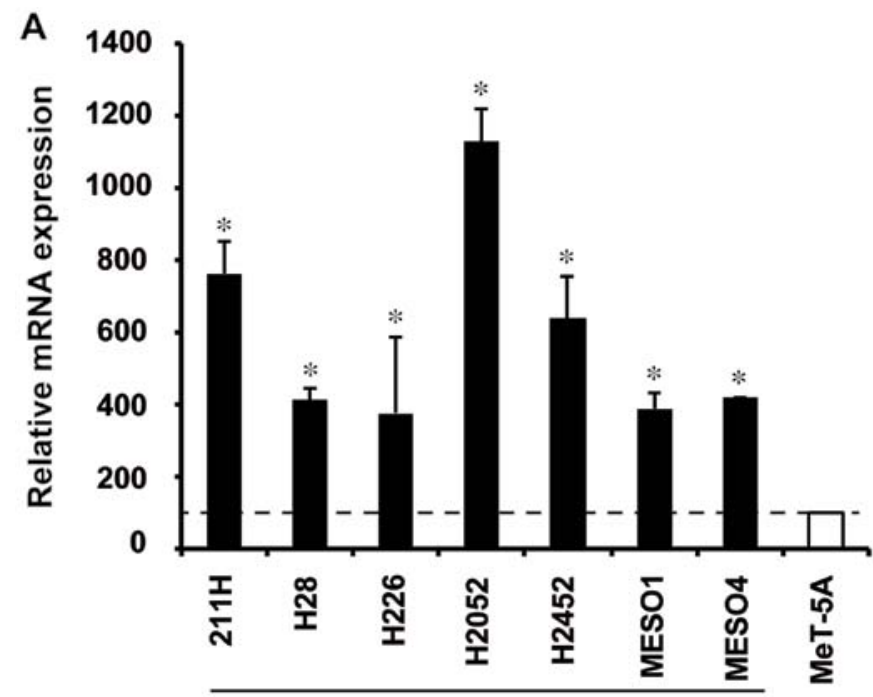

Mesothelioma

\section{B}
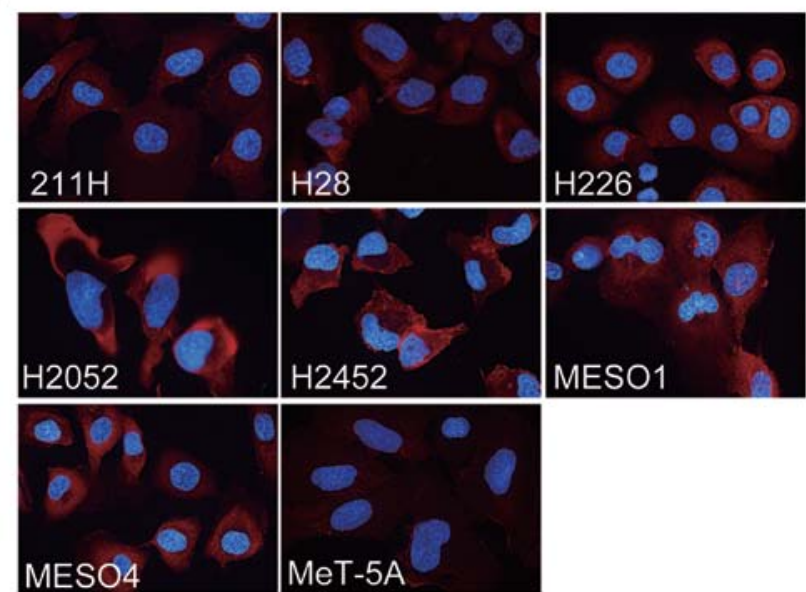

Figure 2. AHNAK expression analysis in cultured cells. (A) AHNAK mRNA expression in seven mesothelioma cell lines and the mesothelial cell line MeT-5A as determined by quantitative real-time RT-PCR is shown. Data represent the mean $\pm \mathrm{SD}(\mathrm{n}=3)$. ${ }^{*} \mathrm{P}<0.05$ and ${ }^{* *} \mathrm{P}<0.01$ versus MeT-5A analyzed by ANOVA with the Dunnett's multiple comparison test. (B) Immunofluorescence staining of mesothelioma and mesothelial cell lines using the anti-AHNAK antibody is shown.

lines was measured by real-time RT-PCR. AHNAK mRNA expression in all seven mesothelioma cell lines $(211 \mathrm{H}, \mathrm{H} 28$, H226, H2052, H2452, MESO1 and MESO4) was significantly higher compared with that in the mesothelial cell line MeT-5A $(\mathrm{P}<0.05)$ (Fig. 2A). AHNAK mRNA levels of mesothelioma cell lines increased by 1.5 - to 5.1-fold in comparison with that of MeT-5A (Fig. 2A), whereas the non-erythroid $\alpha$-spectrin mRNA level of $211 \mathrm{H}$ cells was less than half of MeT-5A (data not shown). By immunofluorescence staining, a high protein expression of AHNAK was observed in all mesothelioma cell lines, and particularly in H2052 cells (Fig. 2B), whereas the AHNAK expression in MeT-5A cells was faint (Fig. 2B). The protein expression levels seemed to be correlated with the mRNA levels.

AHNAK expression analysis in mesothelioma xenograft tumors. To examine the protein expression of AHNAK in
Table II. Summary of immunohistochemical analysis in human specimens.

\begin{tabular}{lrrr}
\hline & No. & Positive $(\%)$ \\
\hline $\begin{array}{lrrr}\text { AHNAK } \\
\text { Sarcomatoid }\end{array}$ & 6 & $6(100)$ \\
$\begin{array}{l}\text { Epithelioid } \\
\text { Biphasic }\end{array}$ & 2 & $2(100)$ \\
Total & 2 & 1 & $(50)$ \\
ERC/mesothelin & 10 & 9 & $(90)$ \\
Sarcomatoid & & & \\
Epithelioid & 6 & 0 & $(0)$ \\
Biphasic & 2 & $2(100)$ \\
Total & 2 & 0 & $(0)$ \\
& 10 & 2 & $(20)$ \\
\hline
\end{tabular}

xenograft tumors, the mesothelioma cell lines were injected subcutaneously into the flank of nude mice. Four cell lines (211H, H226, MESO1 and MESO4) formed tumors at the site of inoculation, but the remaining three cell lines (H28, H2052, and $\mathrm{H} 2452$ ) failed to induce tumor formation. AHNAK protein was expressed in all four xenograft tumors as determined by immunohistochemical staining using the anti-AHNAK antibody (Fig. 3). The cytoplasm and plasma membrane of $211 \mathrm{H}$ tumors was intensely stained with the anti-AHNAK antibody and the plasma membrane of the other xenografts was intensely stained. The epithelioid marker ERC/mesothelin was detected in H226, MESO1 and MESO4 xenografts, but not in $211 \mathrm{H}$. The $211 \mathrm{H}$ xenograft had spindle elongated cell morphology and was negative for ERC/mesothelin, indicating that it formed a tumor with sarcomatoid features. The other xenografts showed glandular and solid epithelial patterns and were positive for ERC/mesothelin, indicating that they formed tumors with epithelioid features.

AHNAK expression analysis in surgical specimens of mesothelioma. We assessed the AHNAK and ERC/mesothelin expression in resected specimens from patients with sarcomatoid, epithelioid, or biphasic mesothelioma by immunohistochemical staining (Fig. 4). In the sarcomatoid specimens, the cytoplasm and plasma membrane of the tumor cells were intensely stained with the anti-AHNAK antibody, but the intensity of the plasma membrane tended to be stronger compared with that of the cytoplasm. ERC/mesothelin expression was not observed. In the epithelioid specimens, strong expression of both AHNAK and ERC/mesothelin was detected on the plasma membrane, but not in the cytoplasm. In one of two biphasic specimens, strong AHNAK expression was observed on the plasma membrane, but not in the other biphasic specimen. ERC/mesothelin expression was not observed in either of the biphasic cases. The summary of immunohistochemical staining in 10 specimens is shown in Table II. The expression of AHNAK was detected in $90 \%$ (9/10) of all mesothelioma specimens: $100 \%(6 / 6)$ of sarcomatoid, $100 \%(2 / 2)$ of epithelioid and $50 \%(1 / 2)$ of biphasic. The ERC/mesothelin expression was detected in $0 \%(0 / 6)$ of 


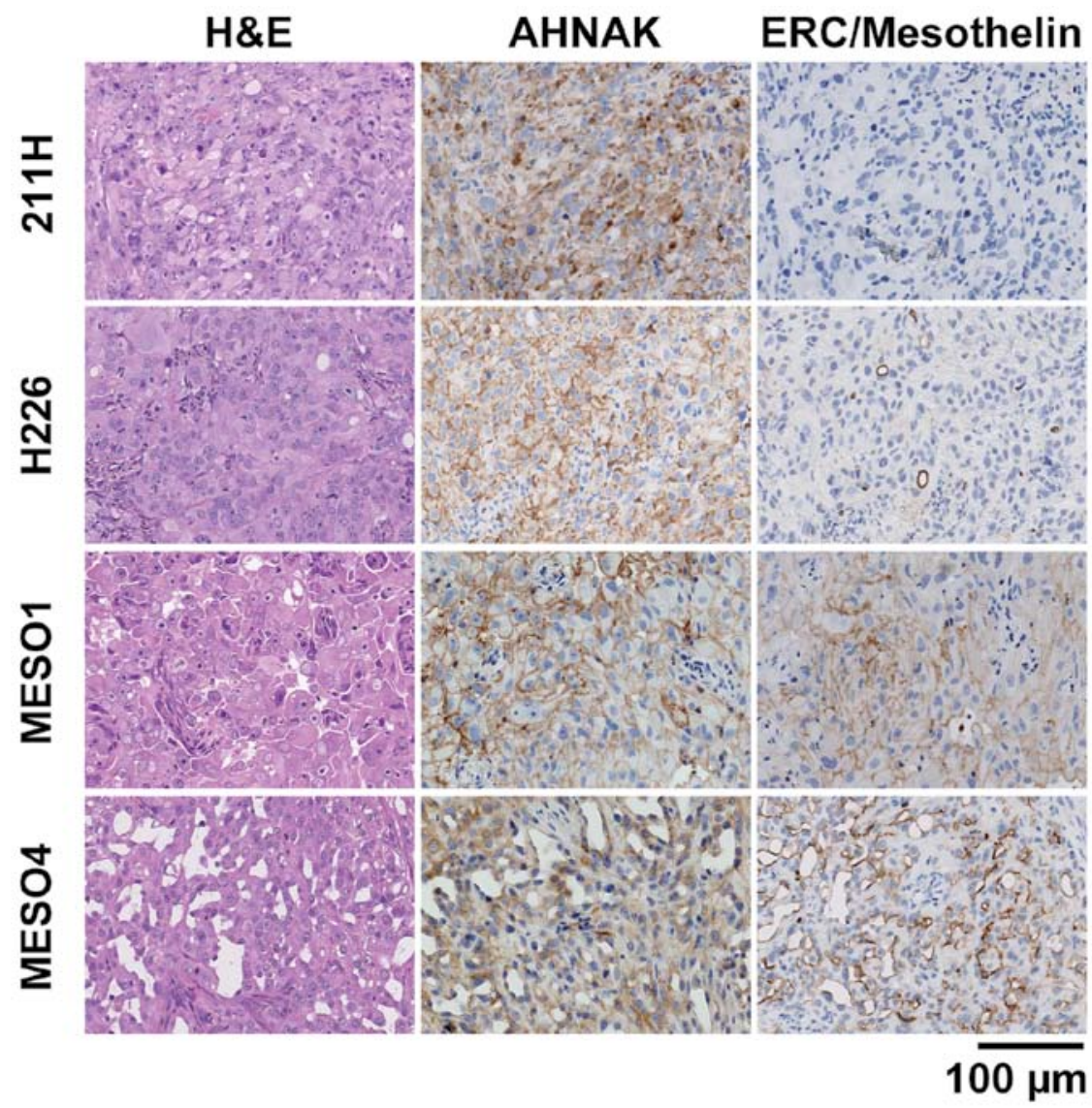

Figure 3. Immunohistological analyses of AHNAK and ERC/mesothelin in xenografts. Representative images of H\&E and immunohistochemical staining of paraffin-embedded tissues using anti-AHNAK and anti-ERC/mesothelin antibodies are shown.
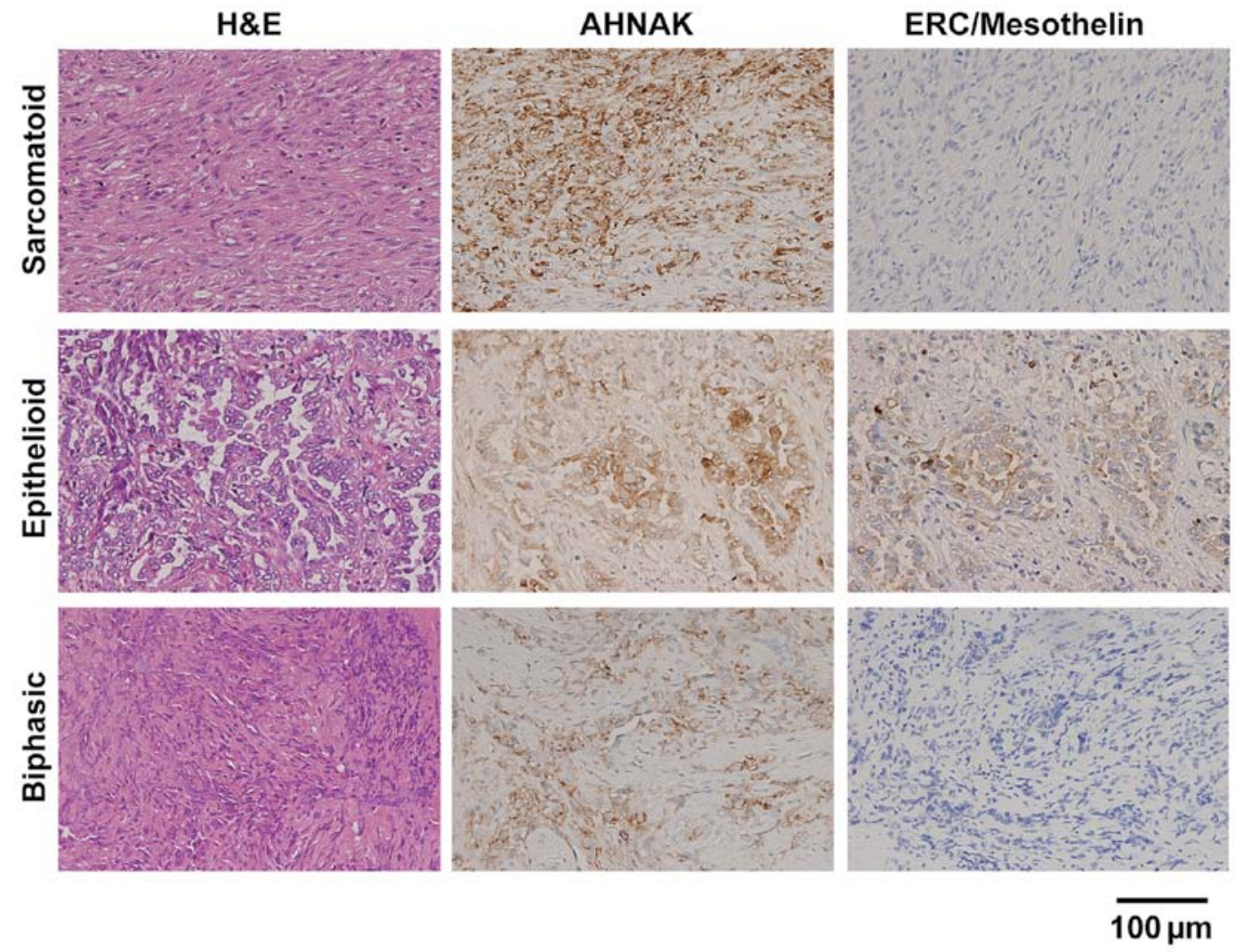

Figure 4. Immunohistological analyses of AHNAK and ERC/mesothelin in human mesothelioma specimens. Representative images are shown of H\&E and immunohistochemical staining of paraffin-embedded tissues using anti-AHNAK and anti-ERC/mesothelin antibodies. 

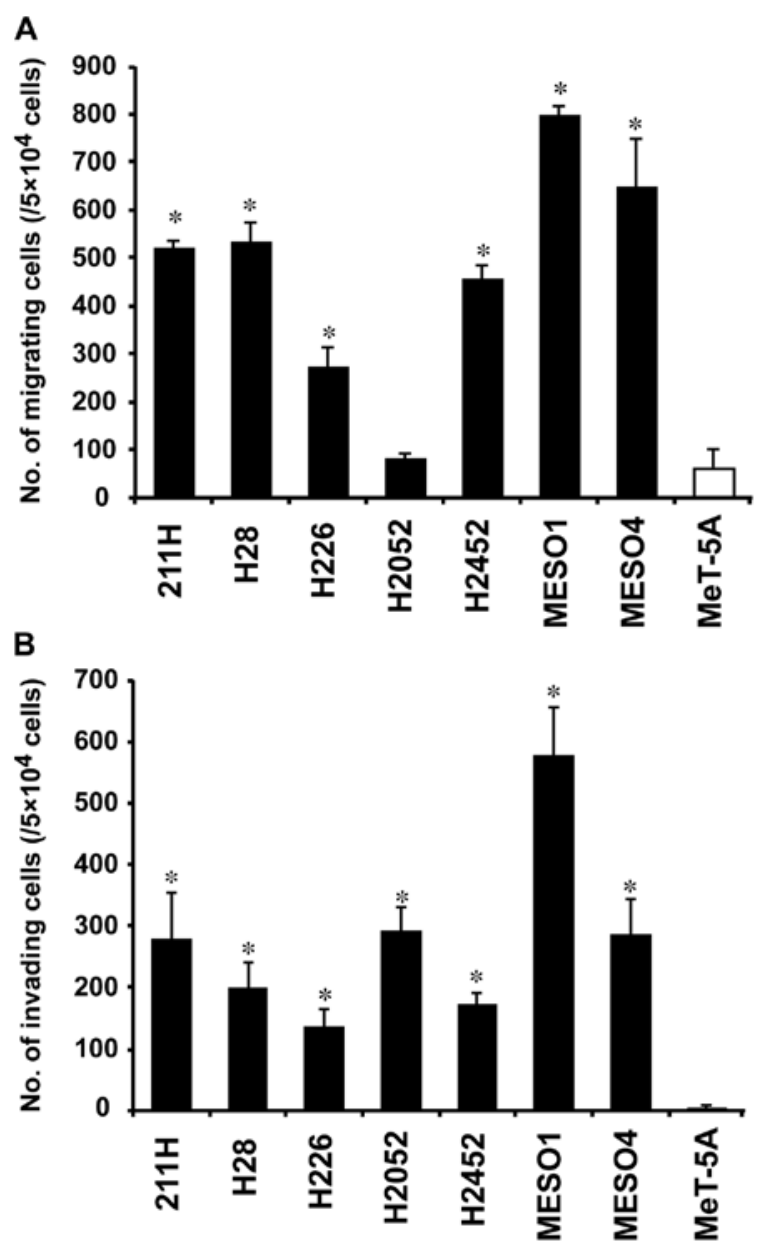

Figure 5. Cell migration and invasion assays in mesothelioma and mesothelial cell lines. Bar graph showing the number of migrating cells (A) and the number of invading cells (B) at $22 \mathrm{~h}$ after seeding. Data represent the mean $\pm \mathrm{SD}(\mathrm{n}=3) .{ }^{*} \mathrm{P}<0.01$ versus MeT-5A (white) as analyzed by ANOVA with the Dunnett's multiple comparison test.

sarcomatoid, $100 \%(2 / 2)$ of epithelioid and $0 \%(0 / 2)$ of biphasic mesothelioma specimens.

Cell migration and invasion assays. We assessed the cell migration and invasion ability of the mesothelioma cell lines. The number of migrating cells in six of the mesothelioma cell lines $(211 \mathrm{H}, \mathrm{H} 28, \mathrm{H} 226, \mathrm{H} 2452, \mathrm{MESO} 1$ and MESO4) was 4.3 - to 12.5 -fold higher than that in MeT-5A $(\mathrm{P}<0.01)$ (Fig. 5A). No significant difference was observed between H2052 and MeT-5A (Fig. 5A). On the other hand, the number of invading cells in all seven mesothelioma cell lines was 33.5to 143.9-fold higher than that in MeT-5A $(\mathrm{P}<0.01)$ (Fig. 5B).

To examine the association of AHNAK and the cell migration or invasion in mesothelioma cells, we conducted migration and invasion assays combined with AHNAK knockdown using the siRNA technique. The knockdown efficiency of the siRNA on AHNAK mRNA expression was measured by real-time RT-PCR at $48 \mathrm{~h}$ after transfection. The AHNAK mRNA expression in AHNAK siRNA-transfected mesothelioma cells was reduced to $<25$ or $23 \%$ compared with that in the mock-transfected cells (without siRNA) or negative control siRNA-transfected cells, respectively (Fig. 6). In six mesothe- lioma cells $(211 \mathrm{H}, \mathrm{H} 28, \mathrm{H} 226, \mathrm{H} 2052, \mathrm{H} 2452$ and MESO4), the number of migrating cells in AHNAK siRNA-transfected cells was decreased to $<21 \%$ compared with that in cells transfected with the negative control siRNA at $48 \mathrm{~h}$ after transfection (Fig. 7A). Although the number of migrating cells in MESO1 transfected with the AHNAK siRNA was decreased to $62 \%$ compared with that in cells transfected with the negative control siRNA, there was a significant difference $(\mathrm{P}<0.01)$ (Fig. 7A). In five mesothelioma cells (211H, H28, H226, H2052 and H2452), the number of invading cells in AHNAK siRNAtransfected cells was reduced to $<26 \%$ compared with that in cells transfected with the negative control siRNA (Fig. 7B). The number of invading cells in MESO1 and MESO4 cells transfected with the AHNAK siRNA was reduced to $<63$ and $46 \%$ compared with that in each negative control siRNA-transfected cell, respectively (Fig. 7B). However, in all cell lines, significant differences were observed between the AHNAK siRNA-transfected and negative control siRNAtransfected cells $(\mathrm{P}<0.05$ in $211 \mathrm{H}, \mathrm{H} 2452$ and MESO1; $\mathrm{P}<0.01$ in H28, H226, H2052 and MESO4) (Fig. 7B).

\section{Discussion}

Malignant mesothelioma is still a very challenging problem because its incidence has been increasing worldwide and its prognosis remains poor (3). Mesothelioma is classified into three histological subtypes (epithelioid, sarcomatoid, and biphasic) by pathological examination including immunohistochemistry. For the epithelioid subtype, the immunohistochemical staining of diagnosis based on pathological markers is well established and achieves an accurate diagnosis, however, to date no adequate maker for the sarcomatoid subtype has been found. There is a critical need to determine a reliable diagnostic marker for sarcomatoid mesothelioma $(7,8)$.

We previously reported that COPA is a candidate protein for use as a pathological maker in the diagnosis of the sarcomatoid subtype of mesothelioma because it is highly expressed in both epithelioid and sarcomatoid mesothelioma models (9). Since there was no anti-COPA antibody suitable for immunohistochemistry, we newly produced and evaluated four antibodies. Unexpectedly, we found that one of these antibodies detected an unknown protein of $120 \mathrm{kDa}$ expressed only in the membrane fraction of the sarcomatoid mesothelioma cell line $211 \mathrm{H}$, and not in the mesothelial cell line MeT-5A. By proteomic analysis of the gel pieces corresponding to the protein band of $120 \mathrm{kDa}$, five proteins (neuroblast differentiation-associated protein AHNAK, nonerythroid $\alpha$-spectrin, Treacher Collins syndrome, pro-ubiquitin and protein BAT2-like 2) were found only in $211 \mathrm{H}$ cells as candidates of the unknown protein. Although AHNAK and non-erythroid $\alpha$-spectrin were matched by multiple peptides, the three other proteins were matched by only one peptide, suggesting that these three proteins were unlikely to be the unknown protein. The non-erythroid $\alpha$-spectrin mRNA level of $211 \mathrm{H}$ cells was half of MeT-5A, in contrast to AHNAK mRNA which was highly expressed in all seven mesothelioma cell lines. These results suggest that AHNAK is the most likely to be the unknown protein. Although the original molecular weight of AHNAK was $680 \mathrm{kDa}(11,12)$, Huang et al reported that AHNAK is cleaved by calpain 3, resulting in the produc- 

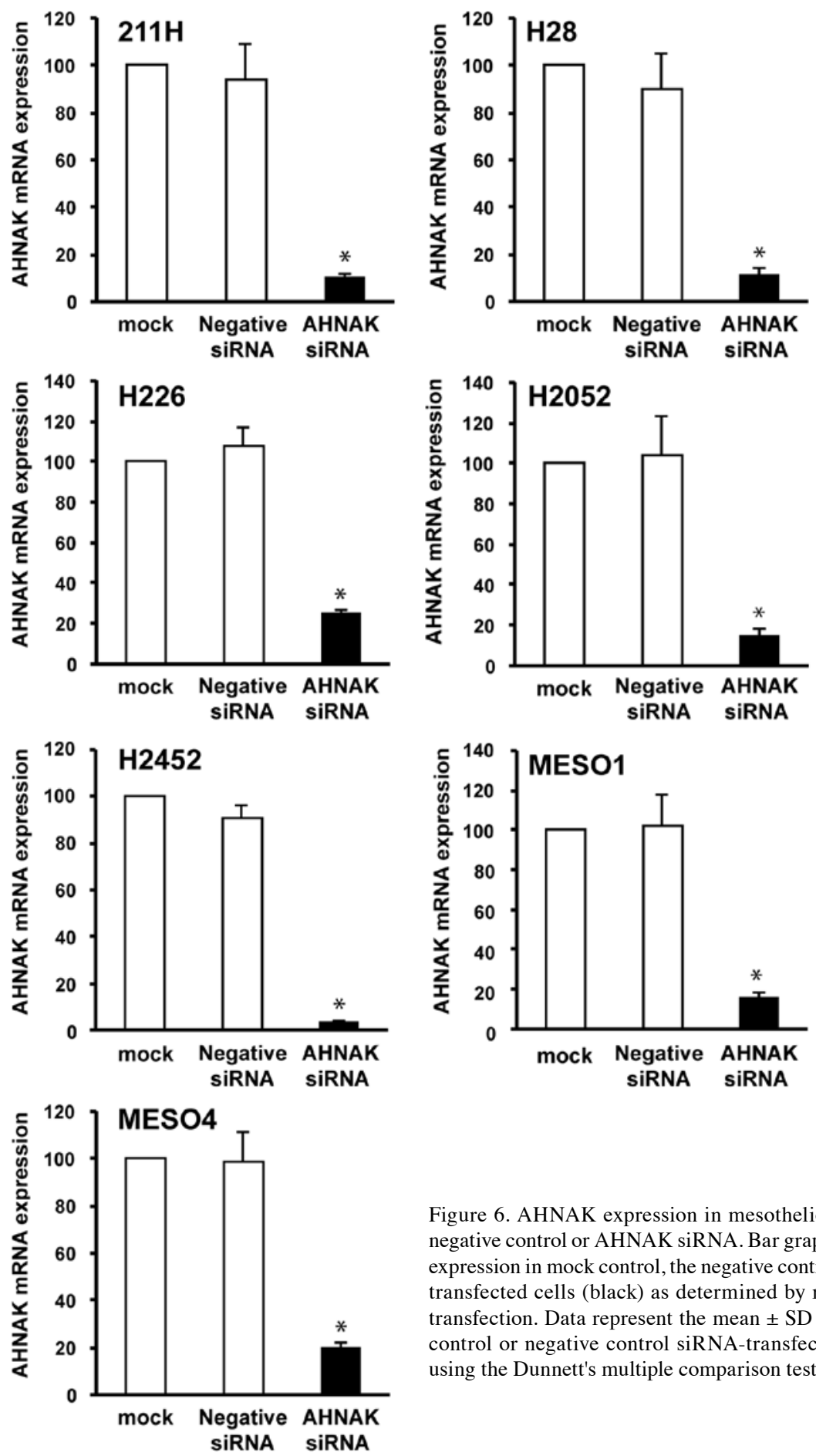

Figure 6. AHNAK expression in mesothelioma cell lines transfected with negative control or AHNAK siRNA. Bar graph showing the AHNAK mRNA expression in mock control, the negative control siRNA-, or AHNAK siRNAtransfected cells (black) as determined by real-time RT-PCR at $48 \mathrm{~h}$ after transfection. Data represent the mean $\pm \mathrm{SD}(\mathrm{n}=3)$. ${ }^{*} \mathrm{P}<0.01$ versus the mock control or negative control siRNA-transfected cells analyzed by ANOVA using the Dunnett's multiple comparison test.

tion of an AHNAK fragment of $120 \mathrm{kDa}$ (13). This fragment size is consistent with the molecular weight of the unknown protein detected by the anti-COPA antibody. Unfortunately, we could not verify whether the AHNAK fragment is the same molecular weight of the unknown protein in the membrane fraction of $211 \mathrm{H}$ cells by western blotting, because we could not find any antibody to work well in western blotting for AHNAK. However, these findings can support the fact that our anti-COPA antibody detected the AHNAK fragment in the membrane fraction of $211 \mathrm{H}$ cells.

We conducted further experiments to evaluate the expression of AHNAK in mesothelioma tissues. Immuno- histochemical staining in xenograft tumors showed that AHNAK was highly expressed in all of four xenografts $211 \mathrm{H}, \mathrm{H} 226$, MESO1 and MESO4 that could form tumors in nude mice. The $211 \mathrm{H}$ xenograft had sarcomatoid features and the others had epithelioid features. These results suggest that AHNAK is expressed in both sarcomatoid and epithelioid mesothelioma. To evaluate the AHNAK protein expression in clinical specimens of mesothelioma, we conducted immunohistochemical analysis of 10 mesothelioma specimens including sarcomatoid, epithelioid and biphasic subtypes. Tumor cells in $90 \%$ of mesothelioma specimens (except for one case with the biphasic subtype) were intensely 

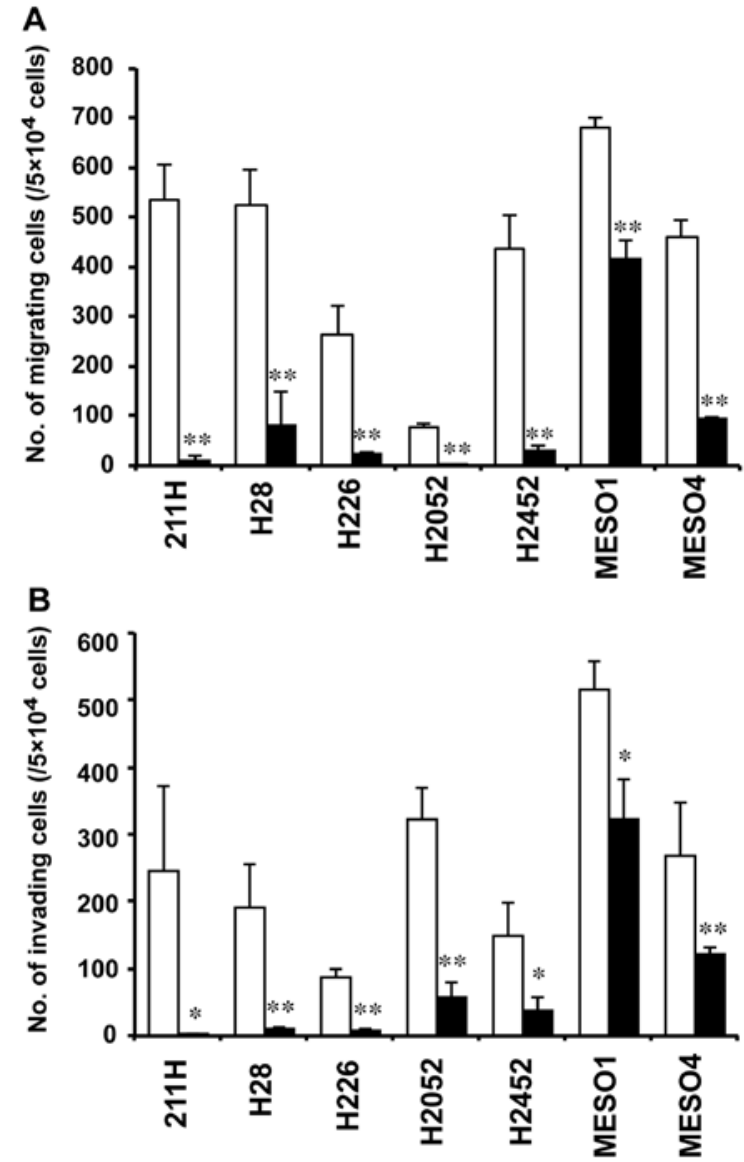

Figure 7. Cell migration and invasion assays in mesothelioma cell lines transfected with negative control or AHNAK siRNA. Bar graph showing the number of migrating cells (A) and the number of invading cells (B) transfected with the negative control (white) or AHNAK (black) siRNA. Data represent the mean $\pm \mathrm{SD}(\mathrm{n}=3) .{ }^{*} \mathrm{P}<0.05$ and ${ }^{* *} \mathrm{P}<0.01$ versus negative control siRNA-transfected cells as analyzed by Student's t-test.

stained with the anti-AHNAK antibody. ERC/mesothelin was expressed in $100 \%$ of the epithelioid cases, but $0 \%$ of the sarcomatoid cases. Although further studies will be necessary to confirm our findings in a larger number of human specimens, the present study suggests that AHNAK has the potential to be a new molecular marker for detecting mesothelioma and that the combined immunohistochemical staining of AHNAK with conventional marker(s) for epithelioid, such as $\mathrm{ERC} /$ mesothelin, could provide helpful information for diagnosing sarcomatoid mesothelioma.

AHNAK is reported to be a membrane scaffold protein (14) and the expression is downregulated in several cell lines derived from neuroblastoma, small cell lung carcinomas and Burkitt lymphomas $(15,16)$; and upregulated in several metastatic tumor cell lines derived from prostate cancer, breast cancer, fibrosarcoma and glioma (17). To our knowledge, the present study is the first showing that AHNAK is highly expressed in mesothelioma. According to a previous study of metastatic tumor cell lines, AHNAK is considered to be one of the essential proteins for pseudopod protrusion and it is involved in tumor cell migration and invasion (17). AHNAK knockdown via RNA interference induced pseudopod retraction, inhibited cell migration and invasion, reduced actin cytoskeleton dynamics, and induced mesenchymal to epithelial transition (17). Many mesothelioma patients receive a diagnosis of local invasion into the endothoracic fascia, mediastinal fat, chest wall or pericardium (18). The local invasion is a major factor preventing surgical resection; therefore, $40 \%$ of mesothelioma patients had unresectable tumors at the time of the initial diagnosis (19). We hypothesized that the high expression of AHNAK is involved in the local invasion of mesothelioma. According to cell migration and invasion assays, six of the seven mesothelioma cell lines showed a higher cell migration rate and all seven mesothelioma cell lines showed increased invasion ability compared with the mesothelial cell line MeT-5A. The AHNAK expression level was not correlated with migration or invasion potency in the seven mesothelioma cell lines. Taken together, AHNAK above a certain expression level might promote cell migration and invasion. The inhibition effect on migration and invasion by AHNAK knockdown in MESO1 cell lines was somewhat lower compared with that in the other lines, suggesting that there could be additional molecule(s) associated with migration and invasion in mesothelioma. Our findings suggest that AHNAK is one of the key molecules of cell migration and invasion in mesothelioma.

In conclusion, we report that the unknown protein of $120 \mathrm{kDa}$ that is expressed only in the membrane fraction of $211 \mathrm{H}$ mesothelioma cells was identified as AHNAK by proteomic and expression analyses. AHNAK was highly expressed not only in xenograft tumors but also in mesothelioma specimens including sarcomatoid, epithelioid, and biphasic subtypes. These results suggest that AHNAK has the potential to be a new marker for detecting mesothelioma, although further clinical investigation is needed. Furthermore, we demonstrated that AHNAK could be involved in the cell migration and invasion of mesothelioma cell lines. Our findings support further clinical investigation to evaluate whether AHNAK expression is associated with metastasis in mesothelioma.

\section{Acknowledgements}

The authors thank Yuriko Ogawa for technical assistance.

\section{References}

1. Robinson BW and Lake RA: Advances in malignant mesothelioma. N Engl J Med 353: 1591-1603, 2005.

2. Tsiouris A and Walesby RK: Malignant pleural mesothelioma: current concepts in treatment. Nat Clin Pract Oncol 4: 344-352, 2007.

3. Kanazawa N, Ioka A, Tsukuma H, Ajiki W and Oshima A: Incidence and survival of mesothelioma in Osaka, Japan. Jpn J Clin Oncol 36: 254-257, 2006.

4. Peto J, Decarli A, La Vecchia C, Levi F and Negri E: The European mesothelioma epidemic. Br J Cancer 79: 666-672, 1999.

5. Milano MT and Zhang H: Malignant pleural mesothelioma: a population-based study of survival. J Thorac Oncol 5: 1841-1848, 2010.

6. Nojiri S, Gemba K, Aoe K, et al: Survival and prognostic factors in malignant pleural mesothelioma: a retrospective study of 314 patients in the west part of Japan. Jpn J Clin Oncol 41: 32-39, 2011.

7. Ordonez NG: Immunohistochemical diagnosis of epithelioid mesothelioma: an update. Arch Pathol Lab Med 129: 1407-1414, 2005. 
8. Sterman DH and Albelda SM: Advances in the diagnosis, evaluation, and management of malignant pleural mesothelioma. Respirology 10: 266-283, 2005.

9. Sudo H, Tsuji AB, Sugyo A, et al: Knockdown of COPA, identified by loss-of-function screen, induces apoptosis and suppresses tumor growth in mesothelioma mouse model. Genomics 95: 210-216, 2010.

10. Ishikawa K, Segawa T, Hagiwara Y, Maeda M, Abe M and Hino O: Establishment of novel $\mathrm{mAb}$ to human ERC/mesothelin useful for study and diagnosis of ERC/mesothelin-expressing cancers. Pathol Int 59: 161-166, 2009.

11. Hieda Y, Tsukita S and Tsukita S: A new high molecular mass protein showing unique localization in desmosomal plaque. J Cell Biol 109: 1511-1518, 1989.

12. Hashimoto T, Amagai M, Parry DA, et al: Desmoyokin, a $680 \mathrm{kDa}$ keratinocyte plasma membrane-associated protein, is homologous to the protein encoded by human gene AHNAK. J Cell Sci 105: 275-286, 1993.

13. Huang Y, de Morree A, van Remoortere A, et al: Calpain 3 is a modulator of the dysferlin protein complex in skeletal muscle. Hum Mol Genet 17: 1855-1866, 2008.
14. Pankonien I, Otto A, Dascal N, Morano I and Haase H: Ahnak1 interaction is affected by phosphorylation of Ser-296 on Cavbeta(2). Biochem Biophys Res Commun 421: 184-189, 2012.

15. Shtivelman E, Cohen FE and Bishop JM: A human gene(AHNAK) encoding an unusually large protein with a 1.2-microns polyionic rod structure. Proc Natl Acad Sci USA 89: 5472-5476, 1992.

16. Shtivelman E and Bishop JM: The human gene AHNAK encodes a large phosphoprotein located primarily in the nucleus. J Cell Biol 120: 625-630, 1993.

17. Shankar J, Messenberg A, Chan J, Underhill TM, Foster LJ and Nabi IR: Pseudopodial actin dynamics control epithelialmesenchymal transition in metastatic cancer cells. Cancer Res 70: 3780-3790, 2010.

18. Servais EL, Colovos C, Rodriguez L, et al: Mesothelin overexpression promotes mesothelioma cell invasion and MMP-9 secretion in an orthotopic mouse model and in epithelioid pleural mesothelioma patients. Clin Cancer Res 18: 2478-2489, 2012.

19. Flores RM, Zakowski M, Venkatraman E, et al: Prognostic factors in the treatment of malignant pleural mesothelioma at a large tertiary referral center. J Thorac Oncol 2: 957-965, 2007. 